\title{
On the subcortical bases of the evolution of language
}

\author{
Philip Lieberman
}

\section{Introduction}

Although most studies on the evolution of the neural bases of human language no longer overtly accept the tenets of phrenology, they implicitly accept the proposition that particular regions of the brain constitute the "seats" of language, thinking, memory, and so on. And since the very beginnings of neuroscience the neural bases of human language and cognition have focussed on the neocortex. In the past forty years, linguists adhering to Noam Chomsky's theories have equated language with syntax, hypothetically specified by an innate, cortical organ, the "Universal Grammar". I shail attempt to shift the focus. I shall show that speech is perhaps the central "unique" aspect of human linguistic ability which also includes lexical and syntactic components, and that all of these elements are learned skills, based on a distributed neural system involving much of the brain, the "Functional Language System" (FLS). Neither the anatomy nor the physiology of the FLS can presently be specified with certainty. However, evidence from many behavioural and neurobiological studies suggests that the neural basis of human language is a distributed network that crucially involves subcortical structures, the basal ganglia. The evolution of functional basal ganglia can be traced back in time to animals similar to present-day frogs (Marin 1999) and their role in the acquisition of learned complex communicative behaviour appears to be similar in other mammalian species as well as in birds (Brainard and Doupe 2000). The evolution of human language, in this light, can be traced back hundreds of millions of years.

However, the particular neural network that regulates human language, the FLS, appears to be a unique, "derived" human characteristic, differentiating humans from closely related species. Like other distributed neural systems that regulate complex behaviour, FLS architecture consists of circuits linking segregated populations of neurons in structures distributed throughout the brain, cortical and subcortical, including the traditional "language" areas (Broca's and Wernicke's areas) as well as other parts of the neocortex. The FLS rapidly integrates sensory information with stored knowledge; it is a dynamic system, enlisting additional neural resources in response to task difficulty. Regions of the frontal lobes of the human 


\section{Philip Lieberman}

neocortex, implicated in abstract reasoning and planning, and other cortical areas are recruited in the FLS as task difficulty increases. The FLS also provides direct access to the information coded in a word, that is, primary auditory, visual, pragmatic, and motor information. The mental operations carried out in the brain are not compartmentalised in fixed modules and the neural bases of human language are intertwined with other aspects of cognition, motor control, and emotion.

No other living species possesses the neural capacity to command spoken language, which serves as a medium both for communication and thought. However, while the human FLS is unique, it appears to have evolved from neural structures and systems that regulate adaptive motor behaviour in other animals. In this light, the subcortical basal ganglia structures usually associated with motor control that are key elements of the FLS reflect its evolutionary history. Natural selection operated on neural mechanisms that yield adaptive - that is, cognitive - motor responses in other species. Given the evolution of the FLS, which as we shall see is reflected in its anatomy and physiology, there is no reason to believe that the basic operations of the human brain differ for motor control and language. Although the neural architecture that regulates motor control and syntax is part of our innate endowment, the details are learned. And the early stages of the evolution of the cortico-striatal neural circuits that regulate human language and thought may have been shaped by Natural Selection to meet the demands of upright bipedal locomotion, the first defining feature of hominid evolution.

\section{Functional neural systems}

The traditional view of the brain bases of language derives from nineteenthcentury phrenology. Phrenological studies claimed that discrete parts of the brain, which could be discerned by examining a person's cranium, were the seats of various aspects of behaviour or character. Neophrenological theorists such as Fodor (1983) do not claim that a bump on your skull shows that you are virtuous, but phrenology lives on. The traditional Broca-Wernicke model of the neural bases of language is in essence phrenological. Paul Broca (1861) ascribed the word-finding difficulties and speech-production deficits of his patient to damage to a frontal region of the neocortex, "Broca's area". In 1874 "receptive" deficits involving comprehension were ascribed to damage to a posterior area of the cortex, "Wernicke's area". Lichtheim's 1885 model, which was adopted without change by Geschwind in 1970, 
claimed that the neural basis of human language was a cortical system linking Wernicke's area with Broca's area. According to this model, Wernicke's area processes incoming speech signals; information is then transmitted via a cortical pathway to Broca's area which serves as the "expressive" language output device. The Broca-Wernicke model is taken by linguists such as Chomsky (1986) and Pinker (1994) to be a valid description of the neural architecture underlying human linguistic ability. According to Pinker, "Genuine language ... is seated in the cerebral cortex, primarily the left perisylvian region", identifying "the human language areas..., Wernicke's and Broca's areas and a band of fibres connecting the two" (Pinker 1994: $334,350)$.

Although the traditional model has the virtue of being simple, neurophysiologic studies show that it is wrong. It is apparent that particular regions of the neocortex are specialised to process particular stimuli, visual or auditory, other regions participate in regulating motor control or emotion or holding information in short-term (working) memory, and so forth. But complex behaviours like reaching for an object or talking are regulated by neural circuits that constitute distributed networks that link activity in many different neuroanatomical structures. As Mesulam notes, "complex. behaviour is mapped at the level of multifocal neural systems rather than specific anatomical sites, giving rise to brain-behaviour relationships that are both localised and distributed" (Mesulam 1990: 598). A given part of the brain typically supports many segregated neuronal populations that project to different parts of the brain forming circuits that regulate different aspects of behaviour.

In other words, although specific operations are performed in particular neuroanatomical structures, overt behaviours are regulated by a network, "functional neural systems", that integrates activity in structures in many regions of the brain. Studies that relate brain activity to behaviour in humans and other species show that a class of functional neural systems exists which generate rapid responses to environmental challenges and opportunities. These neural systems integrate incoming sensory information with stored knowledge and modify or generate goal-directed motor activity to enhance biological fitness. The postulated human FLS regulates language, which sets human beings apart from other living species.

The role of subcortical basal ganglia in the FLS is crucial. Basal ganglia structures form part of neural networks that regulate sequencing in seemingly unrelated activities like moving your fingers (Cunnington et al. 1995), talking (Lieberman et al. 1992; Pickett et al. 1998), comprehending distinctions in 


\section{Philip Lieberman}

meaning conveyed by syntax (Lieberman et al. 1990, 1992; Grossman et al. 1991, 1993; Natsopoulos et al. 1993), and solving cognitive problems (Lange et al. 1992; Pickett et al. 1998). Basal ganglia sequence the pattern generators governing motor activity as well as cognitive operations, by means of segregated neuronal populations in distributed neural networks that project to neuronal populations in other subcortical structures and cortical areas throughout the brain.

\section{The FLS and basal ganglia: cortico-striatal circuits}

The evidence for basal ganglia activity in the FLS is discussed in Lieberman (2000) in some detail. Two sources of evidence are relevant. Since only human beings possess spoken language and complex cognitive behaviour, it is not possible to employ highly invasive techniques that might reveal the neural circuitry of the FLS or the "computations" that are effected in its component neuroanatomical structures. However, our physiology is manifestly similar to that of other species, so valid inferences concerning the human brain can be derived from the study of the brains of other species. Comparative neurophysiologic studies of other species have revealed many aspects of basal ganglia circuitry and function. In some instances, comparable data of human brains using advanced imaging techniques are available. Neurophysiologic studies clearly show that neural circuits link basal ganglia structures and the cerebellum to prefrontal cortical areas implicated in cognition as well as to cortical areas associated with motor control.

Experiments-in-nature dating back to Broca's time constitute the second line of enquiry. Studies of the behavioural effects of brain damage resulting from trauma or disease provide indisputable evidence for the role of subcortical FLS structures. They demonstrate that subcortical structures are essential components of the FLS. Language usually recovers after humans suffer cortical damage, perhaps reflecting cortical plasticity (Elman et al. 1996). However, damage to subcortical circuits results in permanent language deficits (Stuss and Benson 1986). Speech, lexical access, the comprehension of meaning conveyed by sentences, and various aspects of "higher" cognition are regulated by parallel circuits that involve basal ganglia and other subcortical structures as well as other neocortical structures. 
One of the major findings of clinical studies since the 1980 s is that behavioural changes once attributed to frontal lobe cortical dysfunction can be observed in patients having lesions in subcortical basal ganglia. Cummings in his 1993 review article identifies five parallel basal ganglia circuits of the human brain:

a motor circuit originating in the supplementary motor area, an oculomotor circuit with origins in the frontal eye fields, and three circuits originating in prefrontal cortex (dorsolateral prefrontal cortex, lateral orbital cortex and anterior cingulate cortex). The prototypical structure of all circuits is an origin in the frontal lobes, projection to striatal structures (caudate, putamen, and ventral striatum), connections from striatum to globus pallidus and substantia nigra, projections from these two structures to specific thalamic nuclei, and a final link back to the frontal lobe (Cummings 1993: 873).

\section{Representative experimental data: the syntax of rat grooming}

Most linguists believe that the defining characteristic of human linguistic ability is syntax, which links words together into well-formed sentences that can convey an unbounded set of meanings. Studies of rodents show that they too make use of a "syntax", regulated in basal ganglia, to bind individual word-like submovements into well-formed grooming programs. The grooming movements of rats do not convey an unbounded set of meanings - or perhaps any meaning at all. However, experiments show that damage to the striatum of rats disrupts the integrity of the sequences of gestures that normally occur, but does not disrupt the individual gestures that would make up a grooming sequence. In other words, the "syntax" of rat grooming is regulated in the basal ganglia. Damage to other neural structures - prefrontal cortex, primary or secondary motor cortical areas, or cerebellum does not affect the grooming sequence. Electrophysiologic data (Aldridge et al. 1993) show that neuronal activity in basal ganglia regulates the sequence in which these individual movements (forelimb strokes, body licks) occur. 


\section{Philip Lieberman}

\subsection{Learned behaviour}

Rats raised in isolation from other rats execute the same grooming patterns as rats raised in a normal rat environment. Therefore, rodent grooming patterns appear to be coded by a genetically transmitted Universal Grooming Grammar analogous to the Chomskyan Universal Grammar that supposedly determines the syntax of all human languages. Although many linguists accept some form of Chomsky's theory, virtually all developmental studies suggest that human beings learn the particular syntax of the languages that they command by means of cognitive processes and neural mechanisms similar in manner and kind to those employed in learning to play a violin or tennis or to walk (reviews of these studies can be found in Elman et al. 1996; Lieberman 1991, 2000). Neurobiological studies of monkeys and birds reveal the role of basal ganglia in the acquisition of learned behaviour.

The basal ganglia circuits that regulate learned motor tasks in monkeys clearly are shaped by associative processes. Kimura et al. (1993) studied the responses of striatal interneurons as monkeys learned a classic Pavlovian conditioned motor task - the monkeys heard a sound that preceded a task for which they were rewarded. The data showed that neuronal populations in basal ganglia "coded" the learned response. The independent studies of Graybiel and her colleagues (1994) confirm these results. Dopamine-sensitive striatal interneurons (neurons that connect to other neurons within the striatum) responded contingent on reward. The striatal architecture noted by Graybiel et al. (1994) is similar to computer-implemented models of distributed neural networks (Elman et al. 1996) that carry out both associative Hebbian learning and supervised learning. Other independent studies in which monkeys learned tasks when they were rewarded with fruit juice confirm the role of reward-based, "appetitive", activation of midbrain dopamine-sensitive neurons. Interruption of a basal ganglia-forebrain circuit in songbirds results in the birds no longer being able to learn new songs or modify previously learned songs (Brainard and Doupe 2000).

\subsection{Human finger sequencing}

Experiments that indirectly monitor human basal-ganglia activity yield data consistent with these studies of other species. Human basal-ganglia circuits regulate sequential, self-paced, manual motor control tasks. Depleted production of the neurotransmitter dopamine degrades basal ganglia activity 
in Parkinson's Disease (PD), largely sparing cortex (Jellinger 1990). Studies of PD consistently show abnormalities in motor sequencing (Harrington and Haaland 1991). Cunnington and his colleagues (1995) monitored the activity of the supplementary motor area of the cortex in both PD and normal subjects, by means of techniques that used external EEG electrodes to record movement-related potentials: electrical signals in the brain that are emitted before a movement. Subjects pushed buttons with their index fingers in various experimental conditions; signals were recorded from the supplementary motor area before and during each button-push. The buttonpushing data reveal basal ganglia activity similar to that noted by Aldridge and his colleagues for rats as well as studies of spatial sequencing in monkeys that make use of invasive techniques. The basal ganglia:

activate the preparatory phase for the next submovement, thereby switching between components of a motor sequence. Since the basal gangiia and suppiementary motor area are more involved in temporal rather than spatial aspects of serial movement, this internal cueing mechanism would coordinate the switch between motor components at the appropriate time, thus controlling the timing of submovement initiation (Cunnington et al. 1995: 948).

\subsection{Stereotaxic surgery}

Studies of the results of surgery offer another source of data concerning basal ganglia function in human subjects. Stereotaxic surgical techniques, in use for many years, selectively destroy basal ganglia structures such as the internal and external segments of the globus pallidus or the targets of circuits from these structures in the thalamus. Thousands of operations were performed before Levadopa treatment was available to treat PD by offsetting the dopamine depletion that is its immediate cause. These operations often reduced the debilitating rigidity and tremor of PD patients. Marsden and Obeso (1994) review the outcomes of these surgical interventions and similar experimental lesions in monkeys. The seeming paradox is that surgery that destroys subcortical structures, known to regulate various aspects of motor control, has little effect on motor control though it reduces tremor and rigidity. The answer appears to be the distributed parallel nature of the basal ganglia system regulating motor control. Marsden and Obeso note that: 


\section{Philip Lieberman}

neurons in supplementary motor area, motor cortex, putamen and pallidum, all exhibit very similar firing characteristics in relation to movement. For example, populations of neurons in each of these regions appear to code for the direction of limb movement ... and may alter their discharge in preparation for the next movement... Within each of these various motor areas, neuronal populations seem to be active more or less simultaneously, rather than sequentially. They appear to cooperate in an overall distributed system controlling the shape of movement (Marsden and Obeso 1994: 886).

Marsden and Obeso conclude that the basal ganglia have two different motor control functions in human beings.

First, their normal routine activity may promote automatic execution of routine movement by facilitating the desired cortically driven movements and suppressing unwanted muscular activity. Secondly, they may be called into play to interrupt or alter such ongoing action in novel circumstances.... Most of the time they allow and help cortically determined movements to run smoothly. But on occasions, in special contexts, they respond to unusual circumstances to reorder the cortical control of movement (Marsden and Obeso 1994: 889).

But, as many studies show (see Lieberman 2000), the basal ganglia circuitry implicated in motor control does not radically differ from that implicated in cognition:

[T] he role of the basal ganglia in controlling movement must give insight into their other functions, particularly if thought is mental movement without motion. Perhaps the basal ganglia are an elaborate machine, within the overall frontal lobe distributed system, that allows routine thought and action, but which responds to new circumstances to allows a change in direction of ideas and movement. Loss of basal ganglia contribution, such as in Parkinson's disease, thus would lead to inflexibility of mental and motor response... (Marsden and Obeso 1994: 893).

\subsection{Aphasia}

These conclusions are supported by the studies of aphasia that have structured theories of mind and brain since Broca's time. Although the most apparent linguistic deficit of the syndrome named for Broca, "Broca's aphasia", is laboured, slow, slurred speech, other disruptions of normal behaviour can occur such as deficits in fine manual motor control and oral apraxia (Stuss and Benson 1986). Broca's aphasics often also have difficulty exe- 
cuting either oral or manual motor sequences (Kimura 1993). "Higherlevel" linguistic and cognitive deficits also occur. The utterances produced by Broca's aphasics were traditionally described as "telegraphic". When telegrams were a means of communication, the sender paid by the word, and "unnecessary" words were eliminated. Hence the utterances of Englishspeaking aphasics who produce messages like "man eat fish" have the appearance of telegrams. Aphasic telegraphic utterances were thought to be a compensatory behaviour. Aphasic speakers presumably produced short utterances to minimise their speech production difficulties. However, it is evident that Broca's aphasics also have difficulty comprehending distinctions in meaning conveyed by moderately complex syntax. Although agrammatic aphasics are able to judge whether sentences are grammatical with high error rates, these comprehension deficits have been replicated in many independent studies (see Blumstein 1995). Cognitive deficits also occur; Goldstein noted the loss of the "abstract capacity, deficits in planning, and deriving abstract criteria", and "executive capacity generally associated with frontal lobe activity" (Goldstein 1948: 6).

Acoustic analyses show that the characteristic speech production deficit of Broca's syndrome involves impaired sequencing of motor commands. It first is useful to note the aspects of speech production that are intact in Broca's aphasia. The production of the formant frequency patterns that specify vowels and consonants is unimpaired, though there is increased variability (Ryalls 1986). Formant frequency patterns are determined by the configuration of the supralaryngeal vocal tract - primarily tongue and lip activity. We can thus conclude that the control of these structures is unimpaired. The "encoding" or "melding" of formant frequency patterns (Liberman et al. 1967) that characterises the production of human speech is likewise preserved in Broca's aphasia.

\section{Speech encoding}

A short digression on the nature and selective advantage of speech encoding is perhaps germane. Speech encoding is one of the keys to human linguistic ability. It allows us to communicate rapidly, transcending the limits of the human auditory system and the bounds of short-term auditory memory. Formant frequencies are determined solely by the shape and length of the supralaryngeal vocal tract. As a person moves his or her tongue, lips, velum and larynx position, the shape of the vocal tract gradually changes, and so 


\section{Philip Lieberman}

do the formant frequencies. The result is an acoustic melding of the formant patterns that specify "individual" sounds or phonemes into syllable-sized units (Liberman et al. 1967). For example, it is impossible to produce the isolated sound [t] without also producing a vowel or "continuant" such as [ta] or [ts]. The changing formant frequency pattern specifies the initial consonant as well as the vowel: consonant and vowel are fused into a syllable. This process yields the high information transfer rate of human speech because the encoded syllables are transmitted at a rate that does not exceed the fusion frequency of the auditory system. Listeners then resolve the encoded syllables into the phonetic code. The encoding process results in a transmission rate of 20 to 30 phonetic units per second, which exceeds the fusion frequency of the human auditory system. If we were forced to communicate at the slow syllabic rate we generally would forget the beginning of a complex sentence before we heard its end.

The encoding process involves two factors. Inertial left-to-right, "coarticulation" effects inherently encode the formant frequency pattern. For example, when producing the sound [t] of the syllable [ta], the tongue blade must initially be in contact with the palate (the roof of the mouth). The tongue cannot instantly move away from the palate to the lower position necessary for [a]. Consequently, the vocal tract shape gradually changes causing the formant frequency pattern to gradually change as the tongue moves from its syllable-initial position to the [a] position. A similar effect holds for the syllable [tu] except that the consonant "transition" moves into the formant frequency pattern of the vowel $[\mathrm{u}]$. However, inertia cannot account for the "anticipatory" coarticulation typical of speech. Human speakers always plan ahead as they talk, anticipating sounds that will occur. Speakers, for example, "round" their lips (move their lips forward and towards each other) at the very start of the syllable [tu], anticipating the vowel [u]. They don't do this when they produce [ti] because the vowel [i] must be produced without lip-rounding. (It is easy to see this effect if you look into a mirror and say "tea" and "to".) The details of anticipatory planning vary from one language to another, and children learn to produce these encoded articulatory gestures in the first few years of life. Acoustic analyses of anticipatory coarticulation in aphasics show that aphasics do not differ markedly from normal controls (Katz 1988). 


\section{Voice-onset-time}

The defining speech production deficit of Broca's syndrome involves sequencing. Broca's aphasics lose control of the sequencing between larynx and supralaryngeal vocal tract activity. The acoustic cue that differentiates stop consonants such as [b] from [p] in the words bat and pat is voiceonset-time (VOT), the interval between the "burst" of sound that occurs when a speaker's lips open and the onset of periodic phonation produced by the larynx. The sequence between laryngeal phonation and the burst must be regulated to within 20 milliseconds. Broca's aphasics are unable to maintain motor sequencing control; their intended [b]s may be heard as [p]s, [t]s as [d]s, and so on. Control of duration is preserved in Broca's aphasics since the intrinsic duration of vowels is unimpaired.

\section{Prefrontal activity and aphasia}

Although aphasia is by definition a "language" disorder, cognitive deficits occur. In fact, Goldstein, a leading figure in aphasia research, stressed loss of the "abstract" attitude. Goldstein (1948) noted the difficulties that aphasic patients had planning activities and strategies, shifting strategies, formulating abstract categories, and thinking symbolically. Subsequent research has found that these cognitive deficits are associated with impaired frontal lobe, particularly prefrontal, cortical activity (Stuss and Benson 1986). But frontal lobe cognitive deficits do not necessarily result from damage to frontal lobe structures alone. Studies employing Positron Emission Tomography (PET) and CT scans show that damage to elements of stitato-cortical circuits, to either prefrontal cortex, or subcortical structures supporting these circuits, can yield "frontal lobe" cognitive deficits. Metter et al. (1989) found that all of their Broca's patients had damage to the internal capsule and parts of the basal ganglia. PET scans showed that the circuit damage in these patients resulted in vastly reduced metabolic activity in the left prefrontal cortex and Broca's region. 


\section{Philip Lieberman}

\section{The subcortical locus of aphasia}

Marie (1926) claimed that subcortical lesions were implicated in the deficits of aphasia. This was reasonable, since the middle cerebral artery is the blood vessel most susceptible to damage. The central branches of this artery supply the putamen, caudate nucleus and globus pallidus, and one of the central branches of this artery is the thin-walled lenticulostriate artery which is exceedingly vulnerable to rupture. Modern brain imaging techniques confirm Marie's position. It has become apparent that permanent aphasia does not occur, absent subcortical damage. As Stuss and Benson note in their review of studies of aphasia, damage to "the Broca area alone or to its immediate surroundings ... is insufficient to produce the full syndrome of Broca's aphasia.... The full, permanent syndrome (big Broca) invariably indicates larger dominant hemisphere destruction ... deep into the insula and adjacent white matter and possibly including basal ganglia" (Stuss and Benson 1986: 161).

In fact, contrary to the traditional theory, subcortical damage that leaves Broca's area intact can result in Broca-like speech production deficits (Alexander, Naeser, and Palumbo 1987; Mega and Alexander 1994). Damage to the internal capsule (the nerve fibres that connect neocortex to subcortical structures), the putamen, and the caudate nucleus can result in impaired speech and agrammatism similar to that of the classic aphasias in addition to other cognitive deficits. Alexander and his colleagues (1987) reviewed 19 cases of aphasia resulting from lesions in these subcortical structures. Language impairments occurred that ranged from fairly mild disorders in the patient's ability to recall words to "global aphasia" in which the patient produced very limited nonpropositional speech. In general, the severest language deficits occurred in patients who had suffered the most extensive subcortical brain damage. The locus for the brain damage traditionally associated with Wernicke's syndrome includes the posterior region of the left temporal gyrus (Wernicke's area), but often extends to the supramarginal and angular gyrus, again with damage to subcortical white matter below (Damasio 1991). Recent data again show that linguistic capability can be recovered after complete destruction of Wernicke's area (Lieberman 2000). As D'Esposito and Alexander conclude, it is apparent that a "purely cortical lesion - even a macroscopic one - can produce Broca's or Wernicke's aphasia has never been demonstrated" (D'Esposito and Alexander 1995: 41). 


\section{Neurodegenerative diseases}

Studies of the behavioural consequences of diseases such as PD and Progressive Supranuclear Palsy provide evidence for the role of basal ganglia in the FLS. These neurodegenerative diseases result in major damage to basal ganglia, mostly sparing the cortex until the late stages (Jellinger 1990). As Marsden and Obeso (1994) note, tremors, rigidity, and movement disorders occur. However, subcortical diseases also result in linguistic and cognitive deficits that in extreme form constitute a dementia (Albert, Feldman, and Willis 1974).

Sentence comprehension deficits linked to syntax have been noted in independent studies of PD (Lieberman et al. 1990, 1992; Grossman et al. 1991, 1992; Natsopoulos et al. 1994). Illes et al. (1988) found that the sentences produced by PD subjects are generally short and have simplified syntax. Illes and her colleagues attributed these effects to the subjects compensating for speech production difficulties. However, it is clear that the syntactic deficits of PD are not simply a consequence of impaired speech. The data of Lieberman et al. (1990) showed sentence comprehension deficits in PD. The subjects in this experiment simply had to utter the number (one, two, or three) that identified a line drawing that best represented the meaning of the sentence that they heard. Nine out of 40 non-demented PD subjects showed comprehension deficits. The sentence comprehension test that Lieberman et al. (1990) used had been designed for hearing-impaired children. Therefore, the test's vocabulary can easily be comprehended by sixyear-old children, which argues against the subjects having any difficulties with vocabulary. Slight cognitive loss was associated with impaired sentence comprehension; the subjects who had sentence comprehension deficits showed no symptoms of dementia of Alzheimer's type, but cognitive decline was apparent to the neurologist who had observed them over a period of time.

\section{Verbal working memory}

The sentence comprehension deficits of Broca's Aphasia and PD may reflect impairment of "verbal working memory". The concept of working memory derives from research on short-term memory. Short-term memory is usually thought of as a buffer in which information is briefly stored; working memory includes computation as well as storage. In a series of experiments 


\section{Philip Lieberman}

that span thirty years, Baddeley and his colleagues (Gathercole and Baddeley 1993) showed that "verbal working memory" was implicated in both the storage of verbal material and the comprehension of sentences. Verbal working memory involves two components: an "articulatory loop", whereby subjects maintained speech sounds in working memory by subvocally rehearsing them using the brain mechanisms that regulate overt speech, and a "central executive" process.

The central role that speech plays in the FLS is evident in phonetic "rehearsal", whereby words are subvocally maintained in working memory using the neuroanatomical structures that regulate speech production. Many experiments show that subjects have more difficulty recalling a series of longer words than shorter words, as might be predicted if the articulatory buffer had a finite capacity. When articulatory rehearsal is disrupted by having subjects vocalise extraneous interfering words - the numbers one, two, three - during the recall period, recall dramatically deteriorates. Verbal working memory appears to be an integral component - perhaps the key component - of the human functional language system, integrating speech perception, production, semantics, and syntax.

The sentence comprehension test used in the Lieberman et al. (1990, 1992) studies included sentences having syntactic distinctions that are known to place different processing demands in verbal working memory in neurologically intact adult subjects: "centre-embedded" sentences such as The boy who was fat sat down; "right-branching" relative clause sentences such as I saw the boy who is fat; conjunctions like The boy swam and the girl rowed; and "simple" declarative sentences like I saw the boy. Some of the sentences, such as The apple was eaten by the boy, were semantically constrained, others semantically unconstrained (the boy was kissed by the girl.). Whereas control subjects make virtually no errors when they take the test battery used in Lieberman et al. (1990, 1992), the overall error rate was 30 percent for some PD subjects. The subjects' comprehension errors typically involved repeated errors on sentences that had particular syntactic constructions. Therefore, their syntax comprehension errors could not be attributed to general cognitive decline or attention deficits. The highest number of errors ( 40 percent) was made on "left-branching" sentences that departed from the canonical pattern of English having the form subjectverb-object. An example of a left-branching sentence is Because it was raining, the girl played in the house. Thirty percent errors occurred for right-branching sentences with final relative clauses like Mother picked up the baby who is crying. Twenty percent error rates also occurred on long conjoined 
simple sentences like Mother cooked the food and the girl set the table. This again points to verbal working memory load being a factor in sentence comprehension; longer sentences place increased demands on verbal working memory.

Similar sentence comprehension error rates for non-demented PD subjects have been found by Grossman et. al. (1991, 1993) and Natsopoulos et al. (1994) as well as by Pickett et al. (1998) for a subject having brain damage limited to basal ganglia. Grossman et al. (1991) also tested PD subjects' ability to copy unfamiliar sequential manual motor movements (a procedure analogous to that used by Kimura [1993] for Broca's aphasia). The PD subjects' manual sequencing and sentence comprehension deficits were correlated. The correlation is consistent with Broca's area playing a role in manual motor control (Lieberman 1984; Rizzolatti and Arbib 1998) and verbal working memory through circuits supported by basal ganglia (Marsden and Obeso 1994).

\section{Sequencing deficits in speech, syntax, and cognition}

Similarities between the pattern of deficits in PD and Broca's aphasia were found by Lieberman et al. (1992). Acoustic analysis showed a breakdown in nine subjects' VOT control similar in nature to Broca's aphasia. The speech of the PD subjects was similar to that of Broca's aphasics in other ways; they produced appropriate formant frequency patterns and preserved the vowel length distinctions that signal voicing for stop consonants when they occur after vowels. The PD subjects who had VOT overlaps had significantly higher syntax error rates and longer response times on the test of sentence comprehension than the VOT non-overlap subjects; moreover, the number of VOT-timing errors and of syntax errors was highly correlated.

\section{VOT deficits: sequencing or laryngeal control?}

Impaired laryngeal control, usually heard as hoarse, dysarthric speech and low amplitude speech (hypophonia), is a sign of PD. Therefore, it would be reasonable to suppose that impaired control of laryngeal muscles might be the root cause of the VOT deficits of PD. A study of Chinese-speaking PD subjects (Lieberman and Tseng 1994) showed that this was not the case. 
Chinese makes use of "phonemic tones", controlled variations in the fundamental frequency of phonation (F0) which is controlled by the larynx, to differentiate words. The syllable [ma] produced with a level F0 contour in Mandarin Chinese, for example, signifies 'mother', whereas it signifies 'hemp' when produced with a rising F0 contour. Twenty PD subjects read both isolated words and complete sentences in test sessions shortly after or before they took medication that increased dopamine levels. VOT overlap for PD subjects, significantly greater than that of age-matched normalspeaking controls for both the pre-medication and post-medication test sessions, decreased significantly after medication for half of the PD subjects. This showed that dopamine-sensitive subcortical circuits were implicated in sequencing the laryngeal and SVT motor commands that yield VOT distinctions. Acoustic analysis showed that the PD subjects were always able to generate the controlled F0 patterns that specify Chinese phonemic tones. Since the F0 patterns that specify these phonemic tones are generated by precise laryngeal manoeuvres, it is apparent that sequencing deficits are responsible for the observed VOT overlaps.

In short, the VOT overlaps that can occur in PD appear to reflect impaired sequencing of the individual motor commands that constitute the motor programs that generate speech. This is not surprising in the light of studies of sequential motor activity in PD (Marsden and Obeso 1994; Cunnington et al. 1995), and the sequencing of "submovements" of rodent grooming noted by Aldridge et al. (1993). If the human neural circuitry regulating voluntary laryngeal activity during speech production is similar to that of monkeys, then the locus of VOT sequencing deficits may be the coordination of the activity of two independent neural circuits: a laryngeal circuit involving anterior cingulate gyrus, and independent circuits involving neocortical areas that regulate supralaryngeal vocal tract manoeuvres. Significantly, no neocortical areas appear to be implicated in the regulation of nonhuman primate vocalisations (Sutton and Jurgens 1988).

Discussions of other experimental data consistent with basal ganglia regulating and shifting sequential motor and cognitive acts are noted in Lieberman (2000). Hypoxia on Mount Everest resulting from exposure to altitude results in VOT and sentence comprehension deficits (Lieberman et al. 1994, 1995). Pickett et al. (1998), in a study of a subject having brain damage limited to basal ganglia, found speech motor sequencing deficits as well as deficits involving sequencing in the comprehension of distinctions in meaning conveyed by syntax and in cognitive tasks. Vargha Khadem et al. (1998) documented speech and language defieits in the alleged "language 
gene" family (misrepresented in Pinker 1994): subjects who have a genetically transmitted anomaly that results in bilateral reduction of caudate nucleus volume. Cognitive deficits similar to those occurring with frontal lobe damage can be traced to impaired basal ganglia activity in PD (Lange et al. 1992). Cerebellar damage also yields similar deficits (Pickett 1998). It is clear that subcortical structures are critical elements of the FLS that regulates human language and some aspects of cognition.

\section{On the evolution of adaptive behaviour}

Despite somewhat strident claims to the contrary (Pinker 1994), languagetrained chimpanzees produce about 150 words using manual sign language or computer keyboards, roughly equivalent to the abilities of two year-old children. These chimpanzees also can understand spoken English words (Gardner and Gardner 1984; Gardner et al. 1989; Savage-Rumbaugh and Rumbaugh 1993). Indeed, other species comprehend spoken words. Pet dogs almost always comprehend a few words, and the circus dog "Fellow" understood at least 50 words (Warden and Warner 1928). Therefore, lexical ability itself, dissociated from speech production, is a primitive feature of language and undoubtedly existed in the ancestral species that was the common ancestor of human beings and apes as well as all archaic hominid lineages. Syntactic ability, which generally is taken by linguists (e.g., Pinker 1994; Calvin and Bickerton 2000) to be a unique human attribute, is present to a limited degree in language-trained chimpanzees. Analyses of the American Sign Language used by the Project Washoe chimpanzees show that they used two-sign combinations and some three-sign combinations. Savage-Rumbaugh et al. (1986) demonstrate that the six-year-old pygmy chimpanzee Kanzi comprehends simple sentences having the canonical form of English in which the subject always proceeds the object. Kanzi responded correctly about 75 percent of the time when he heard sentences like: Put the pine needles on the ball. And: Put the ball on the pine needles. Kanzi appropriately placed the pine needles on the ball or the ball on the pine needles in response to English-language commands, demonstrating a sensitivity to basic word order in English.

The sole aspect of human linguistic ability that chimpanzees lack is speech. Despite attempts spanning the past 300 years, no one has been able to train a chimpanzee to talk. The supralaryngeal vocal tract anatomy of chimpanzees prevents them from producing vowels such as [i] and [ $\mathrm{u}$ ] (the 


\section{Philip Lieberman}

vowels of the words see and do). However, speech communication could take place without producing the vowel [i], albeit with increased error rates (see Lieberman 1984, 1991, 2000). Acoustic analyses of chimpanzee vocalisations and computer modelling of the range of sounds that their vocal tracts can produce show that ape anatomy would permit them to speak producing bilabial and "alveolar-dental" consonants like [b], [p], [m], [t], [d], [s], and so on as well as all vowels save [i], [u] and [a] (Lieberman 1968, 1984). But chimpanzees cannot even freely permute the sounds that occur in their natural repertoire of calls. The factor limiting chimpanzee speech is the chimpanzee brain. Chimpanzees cannot produce vocalisations that are not bound to specific emotional states (Lieberman 1994a): "Chimpanzee vocalisations are closely bound to emotion. The production of a sound in the absence of the appropriate emotional state seems to be an almost impossible task for a chimpanzee"(Goodall 1986: 125). Human beings are able to freely permute the motor commands, the "submovements" that constitute the sounds of human speech to form words. The absence of productive speech and the limited cognitive and syntactic abilities of apes and other species may reflect their basal ganglia circuitry. It is quite possible that our human qualities, our ability to unbind articulatory submovements to generate a virtually unlimited number of words, regulate complex syntax, and abstract thought, derive from some unique aspects of human cortico-striatal circuitry. Further study using noninvasive techniques that will allow us to map the detailed striatal circuits of humans and apes will answer this question.

\section{The antiquity of hominid speech}

The probable absence of a modern human vocal tract in Neanderthals and its almost certain absence in Australopithecines and Erectus hominids (Lieberman 1984), does not indicate the complete absence of speech. As the initial Lieberman and Crelin (1971) paper on Neanderthal speech capabilities carefully pointed out, Neanderthals undoubtedly possessed speech, albeit less efficient speech than modern humans. This conclusion follows from the logic of natural selection. The perceptual "decoding" of the formant frequency patterns that convey particular speech sounds must take account of the length of a speaker's vocal tract (longer vocal tracts produce lower formant frequencies for the "same" speech sound than shorter vocal tracts). The vowel [i] is optimal for this process (Nearey 1979). It is possible to perceive speech without [i], but its presence makes speech perception less 
susceptible to error. However, the human vocal tract increases the risk of choking to death when we swallow solid food (noted by Charles Darwin) and has other negative consequences (increased risk of death due to impacted teeth and less efficient chewing). Therefore, the restructuring of the hominid SVT to enhance speech perception would not have contributed to biological fitness unless speech and language were already present in the hominid species ancestral to modern Homo sapiens. There otherwise would have been no reason for the retention of the lower laryngeal position of the human SVT. In short, we can conclude that speech and some form of language (including syntactic ability, present in rudimentary form in living apes) must have already been present in Neanderthals and in the common ancestors of Neanderthals and modern humans, Homo erectus and perhaps Australopithecines.

Indeed, it has become clear that some aspects of human speech are very primitive in an evolutionary sense. The neural processing that allows us to determine the length of the SVT of the person to whom we are listening occurs in many other species; studies of monkeys suggest that they also can judge the length of another monkey's vocal tract, which is a good index of a monkey's size, using a similar process (Fitch 1997). Early hominids surely must have possessed this ability. Nonhuman primate calls also can be differentiated through formant frequency patterns generated when monkeys or apes close or open their lips as they phonate (Lieberman 1968). The fundamental FO, which is determined by laryngeal muscles and alveolar (lung) air pressure, is one of the principal cues that signals the end of a sentence and major syntactic units. Most human languages make use of controlled variations of F0 to produce "tones" that differentiate words. Since apes possess laryngeal anatomy that can generate F0 contours, early hominids must have had this ability. The roots of speech communication may extend back to the earliest phases of hominid evolution.

\section{Walking and basal ganglia}

About 5 million years ago a species lived that was the common ancestor of present day apes and humans. The fossil remains of early hominid species, like those of the 4.4 million-year-old Ardipithecus ramidus, resemble apes who could have walked upright (White et al. 1994). It is quite possible that upright bipedal locomotion may have been the preadaptive factor that selected for neural mechanisms that enhanced motor ability (Jesse Hoehstadt, 
personal communication). Human beings learn to walk. The "walkingreflex" that exists in new-born human infants is controlled by a quadripedal neural "pattern-generator" that reflects our hominoid ancestry. Heel strike, which marks efficient bipedal locomotion, takes toddlers several years to develop (Thelen 1984). The subcortical basal ganglia structures of the FLS regulate upright, bipedal locomotion. One of the primary signs of $\mathrm{PD}$, in which basal ganglia circuits are degraded, is impaired locomotion. Thus, upright, bipedal locomotion may have been the initial selective force for the enhancement of the subcortical-cortical circuits that regulate sequencing of both motor and cognitive acts. Careful developmental studies relating the onset of walking to language, cognition, and brain development in young children will show whether this suggestion has any merit.

Many topics relating to the subcortical brain and the evolution of language are discussed in Lieberman (2000). The dopamine depletion that characterises PD may also directly reduce verbal working memory span. Ongoing research suggests that this effect can occur independent of sequencing deficits. Studies of the neural bases of motor control also show that complex behaviours are generally learned rather than innate. Since the same subcortical neuroanatomical structures participate in both motor control and language, it is likely that syntax is learned rather than specified by a Universal Grammar. Neurophysiologic studies also show that algorithmic descriptions of motor behaviour are at best metaphors; the neural activity that governs motor control is parallel and distributed. Since there is no evidence that the physiology of the brain differs in any fundamental manner for motor control and language, we must conclude that algorithmic descriptions of language processing have the same status as they do for motor control. In short, the cumbersome and inadequate "rules of grammar" - innate Universal Grammar specifying the "rules" of syntax proposed by Chomsky and his disciples - are biologically implausible. 